\title{
CONTROLE INTEGRADO DE PROCESSO E PRODUTO PARA GESTÃO DA QUALIDADE NA LAMINAÇÃO A QUENTE DA VOTORANTIM SIDERURGIA *
}

\author{
Izabelle Lannes Salgueiro Ferreira ${ }^{1}$ \\ Raphael Barbosa de Souza ${ }^{2}$ \\ José Carlos da Silva ${ }^{3}$ \\ Gabrielle Cristine Lemos Duarte Freitas ${ }^{4}$
}

\section{Resumo}

O presente trabalho tem como objetivo mostrar o Programa da Qualidade desenvolvido na Gerência de Laminação a Quente da Votorantim para aperfeiçoar o sistema de gestão da qualidade. Tendo como base a melhoria no método de análise dos parâmetros de processo e produto, foi identificada a oportunidade de desenvolver um modelo integrado com a finalidade de coletar resultados dos parâmetros produtivos de forma instantânea, obtendo o controle online do processo e produto. A implantação do ProQuali possibilitou a realização de análises de campanha para produtos críticos pela equipe técnica e auxiliou a equipe operacional na tomada de decisão online durante o processo. Com isto foi possível evidenciar uma evolução significativa nos principais indicadores do laminador: índice de rendimento metálico (RM), utilização (IU) e geração de produtos não conforme (NC).

Palavras-chave: Gestão da Qualidade; Processos; Variáveis.

\section{INTEGRATED PROCESS AND PRODUCT CONTROL FOR QUALITY MANAGEMENT IN THE HOT ROLLING AT VOTORANTIM}

\begin{abstract}
The proposed work aims to show the Quality Program developed at Votorantim Hot Rolling to improve the quality management system. Based on the improvement in the method of analysis of process parameters, the opportunity was identified to insert into the process an integrated model with the purpose of collecting results of the productive parameters in an instantaneous way, obtaining the online control of the process and product.The implementation of ProQuali made it possible to carry out campaign analyzes for critical products by the technical team and assisted the operational team in making online decisions during the process. With this, it was possible to show a significant evolution in the main indicators of the rolling mill: metallic income index (MRI), utilization (UI) and generation of nonconforming products (NC).
\end{abstract}

Keywords: Quality management; Process; Variables.

1 Engenharia Metalúrgica UFF, Coordenadora de Melhoria, Gerência de Laminação a Quente, Votorantim Siderurgia, Barra Mansa, Rio de Janeiro, Brasil.

2 Engenharia de Produção UniFOA, Gerência de Laminação a Quente, Votorantim Siderurgia, Barra Mansa, Rio de Janeiro, Brasil.

3 Técnico Mecânica, Técnico Especialista de Processo, Gerência de Laminação a Quente, Votorantim Siderurgia, Barra Mansa, Rio de Janeiro, Brasil.

4 Engenharia Metalúrgica UFF, Estagiária Nível Superior, Gerência de Laminação a Quente, Votorantim Siderurgia, Barra Mansa, Rio de Janeiro, Brasil. 


\section{INTRODUÇÃO}

Mercados cada vez mais dinâmicos e competitivos necessitam de um sistema de gestão da qualidade para o gerenciamento da expressiva massa de dados que são gerados pelos processos industriais.

Segundo o Handbook of Industrial Engineering Technology and Operations Managemen [1], um sistema que envolva a área de engenharia, tanto a de produto como a de processo, é capaz de otimizar análises, avaliar dados do processo e auxiliar na tomada de decisão com modelos de predições estabelecidos.

Um sistema de gestão bem estruturado ajuda a equipe técnica da empresa a otimizar respostas aos problemas diários que surgem nos processos, muitas vezes associado devido a variações em uma ou mais variáveis de controle, como ocorre no caso do processo de laminação de vergalhões e perfis.

O aperfeiçoamento dos sistemas de gestão da qualidade nas organizações é um fator importante, visto que estamos nos adaptando a constante mudanças, quer seja para atender às normas técnicas ou para alguma alteração no processo.

A melhoria contínua foi definida como um processo de inovação incremental, focada e contínua envolvendo toda a equipe [2]. O primeiro passo para a busca da melhoria contínua é a implantação de um sistema da qualidade [3].

Com base nestes conceitos, foi desenvolvido o Programa da Qualidade (ProQuali) um projeto piloto dentro da Gerência da Laminação a Quente da Votorantim Siderurgia. O ProQuali é um modelo de gestão que integra as informações das etapas do processo produtivo, controle e inspeção online, laboratório de ensaios mecânicos e acabamento; ou seja, organiza e gerencia os dados e informações dessas etapas mapeadas no processo.

Através da utilização do ProQuali foi possível: implementar uma rotina de análises de campanhas para os produtos críticos pela equipe técnica, auxiliar a equipe operacional na tomada de decisão online durante o processo e evidenciar evolução nos principais indicadores do laminador.

Esta evolução dos indicadores foi muito significativa para o produto de maior criticidade do Laminador, a barra redonda laminada,

\section{MATERIAIS E MÉTODOS}

A metodologia utilizada neste projeto foi PDCA (do inglês: PLAN-DO-CHECK - ACT) que é um método interativo de gestão de quatro passos, utilizado para o controle e melhoria contínua de processos e produtos (planejamento, execução, verificação e padronização), conforme representado na figura 1:

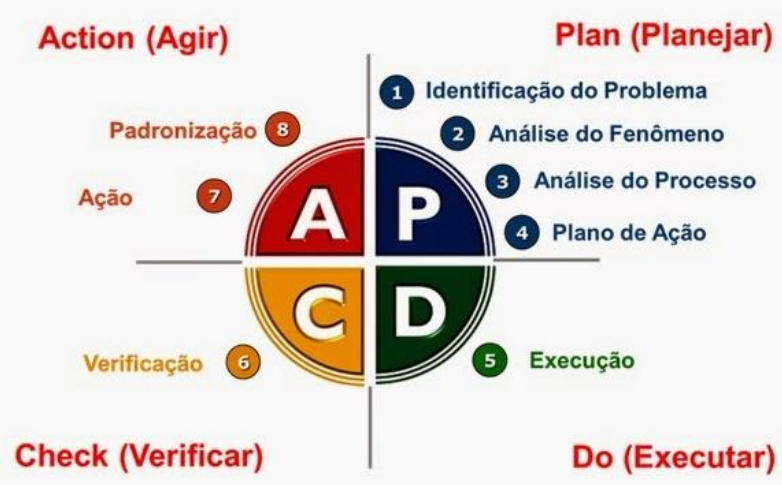

Figura 1. Ciclo PDCA 


\subsection{Planejamento}

$\mathrm{Na}$ etapa de planejamento, identificamos que os dados do processo não estavam colaborando para uma gestão da qualidade eficaz e eficiente. O modelo existente até então não suportava a necessidade de análises de campanhas e um controle online do processo devido esses dados coletados pelos operadores e inspetores não serem armazenados de forma informatizada. O modelo utilizado não permitia uma análise dinâmica e frequente, o que impedia um controle mais eficiente das variáveis críticas do processo. A equipe do projeto verificou que cosumia-se horas para organizar, tratar e analisar os dados dessas variáveis; reduzindo o tempo necessário para tomar decisões e atuar no processo.

Após validar o projeto e informatizar essas variáveis, definimos a necessidade de:

- Um programa integrando todas as variáveis críticas do processo e produto e armazenar os dados em um banco de dados. Esse programa foi denominado ProQuali v01.2016, responsável por organizar todos os dados inseridos pelos operadores chaves (postos 1, 2, 3 e 4 da figura 2).

- Um programa de análise estatística que auxilie a equipe técnica na tomada de decisão e a equipe operacional no controle do processo. Esse programa foi denominado STATPro v01.2017, responsável pelas análises e controle online do processo para as equipes técnica e operacional.

Após revisão do processo, o planejamento do ProQuali foi feito com base nos pontos críticos mapeados pela equipe, conforme observa-se na figura 2 :

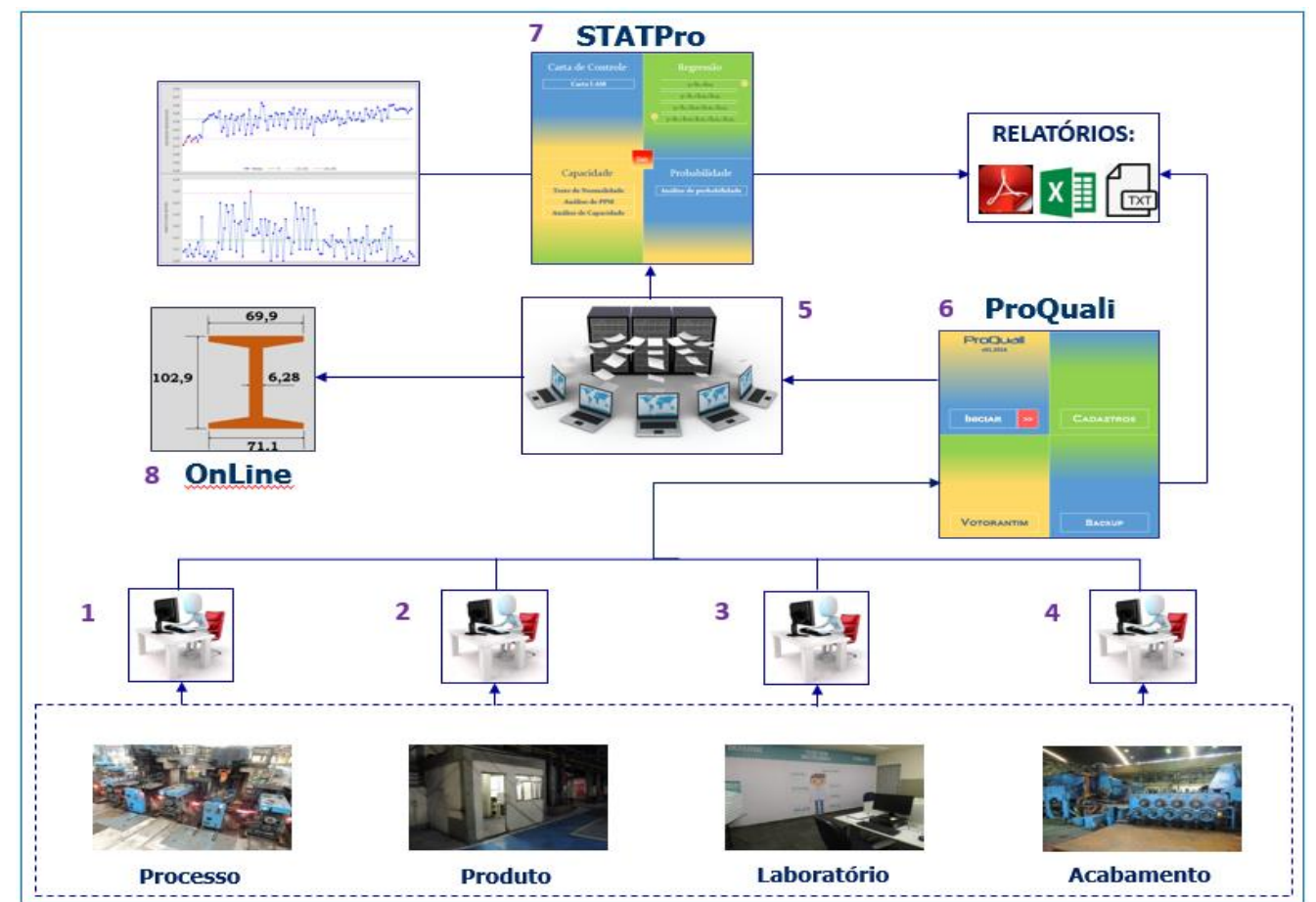

Figura 2. Fluxo de desenvolvimento do ProQuali. 
As ações para o modelo proposto foram:

- Mapear os postos críticos do laminador. Conforme representado na Figura 2, os postos (1, 2, 3 e 4) foram estabelecidos de acordo com o Mapa do Processo da Gerência de Laminação a Quente;

- Definir e construir os relacionamentos entre as variáveis do processo e produto que seriam armazenadas no banco de dados;

- Desenvolver um Sistema de Gestão (ProQuali v01.2016);

- Desenvolver um Sistema de Análises (STATPro v01.2017);

- Desenvolver um Sistema de Controle OnLine do Processo (STATPro v01.2017).

\subsection{Execução}

Como foi considerado um projeto piloto na Votorantim, valorizamos a perspectiva técnica e financeira para o desenvolvimento do ProQuali. Tanto a propriedade intelectual como o software necessários para o desenvolvimento do sistema de gestão estava disponível para a equipe de trabalho e, portanto, o custo inicial do Programa da Qualidade foi zero; necessitando apenas de um planejamento em relação à agenda de cada membro da equipe.

A base para o ProQuali foi o Visual Basic for Applications (VBA) da Microsoft. O VBA é uma implementação do Visual Basic da Microsoft incorporada em todos os programas do Microsoft Office (Word, Power Point, Excel, Access) e outras aplicações como o Visio. Os recursos avançados de formulários, automação e controle de máquinas do VBA permitiram ao programa ser robusto e totalmente rápido para administrar o cadastro dos dados em um banco de dados.

Durante a programação, as principais entregas mapeadas para o ProQuali foram:

- Facilidade e agilidade no acesso de dados;

- Controle e segurança dos dados;

- Interface amigável;

- Relatórios detalhados e otimizados;

- Gráficos interativos e de fácil compreensão;

- Análises estatísticas para tomada de decisão;

- Controle online do processo.

A figura 3 representa a tela de acesso do ProQuali v0.2016. 


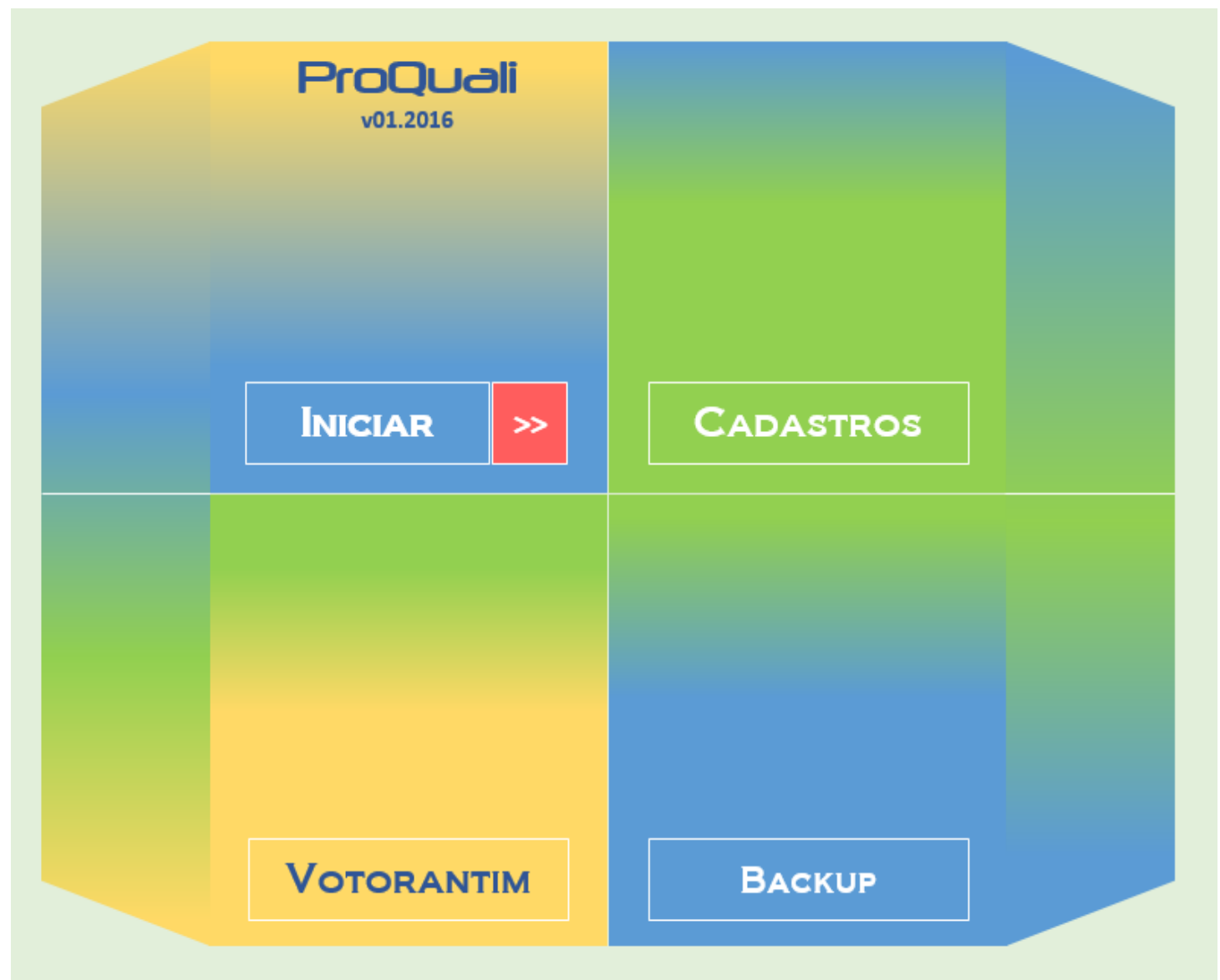

Figura 3. Tela de acesso do ProQuali.

Com base no Mapa de Processo do Laminador, definimos como variáveis críticas: dimensional do material nos passes do laminador (trem desbastador, trem intermediário e trem acabador), temperatura da barra e tonelagem dos canais em cada passe.

A figura 4 observa-se o formulário de cadastro para o processo. 


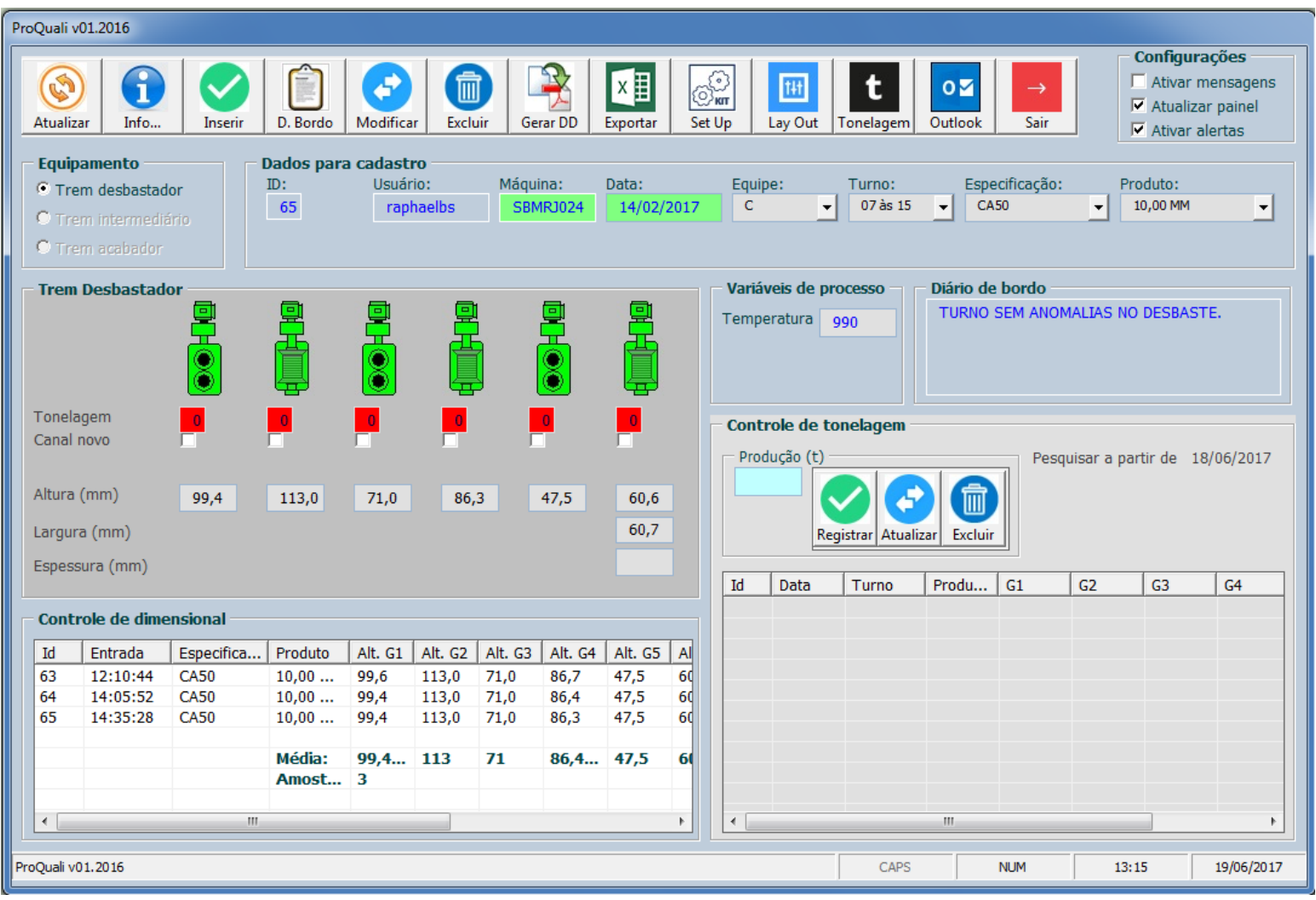

Figura 4. Formulário para cadastro das variáveis de controle do processo.

No formulário do inspetor de qualidade (posto 2 conforme a figura 2), inserimos as variáveis de cada produto conforme a Norma Técnica NBR 7480 específica estabelece.

Na figura 5 observa-se o formulário de cadastro para as variáveis do produto.

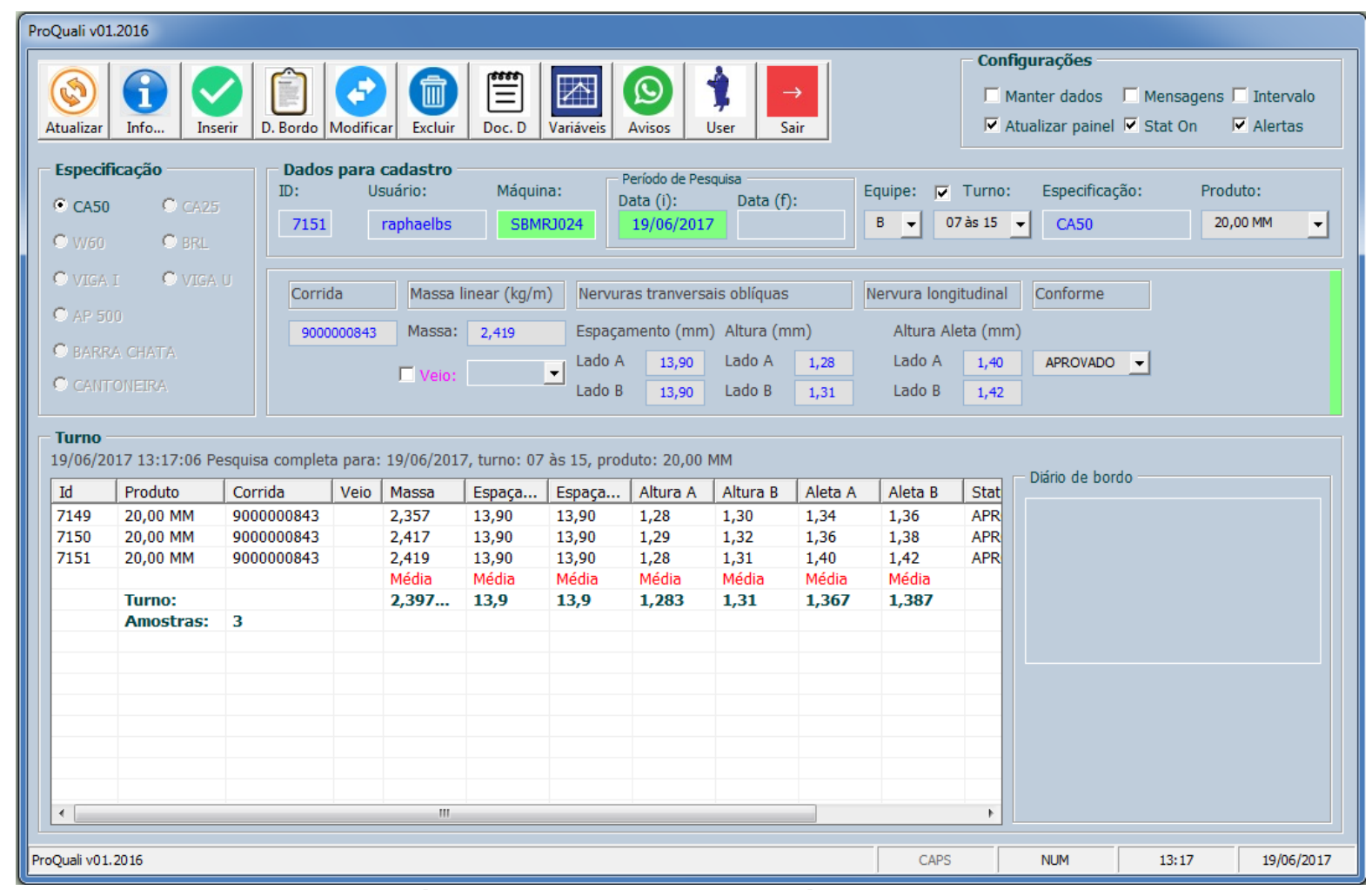

Figura 5. Formulário para cadastro das variáveis de controle do produto. 
Todos os resultados dos ensaios foram cadastrados no formulário de ensaios mecânicos do ProQuali v01.2016, conforme representado na figura 6. Esse cadastro ajudou a desenvolver relacionamentos mais dinâmicos entre as variáveis do processo e produto.

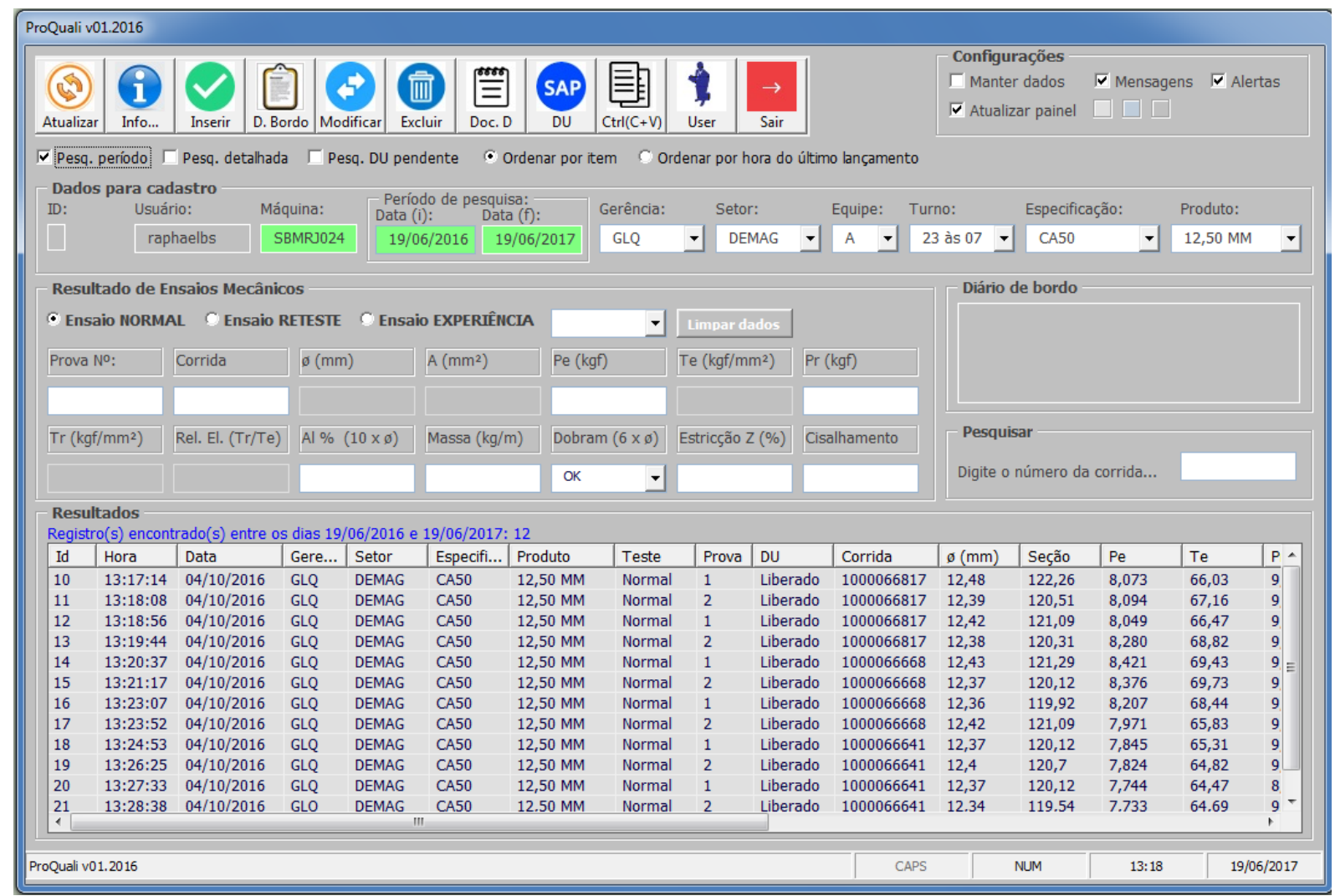

Figura 6. Formulário para cadastro dos resultados do Ensaios Mecânicos.

O formulário a seguir, apresenta a tela do operador da endireitadeira (posto de trabalho 4 conforme a figura 2), com as variáveis de ajuste axial e radial dos rolos endireitadores. 


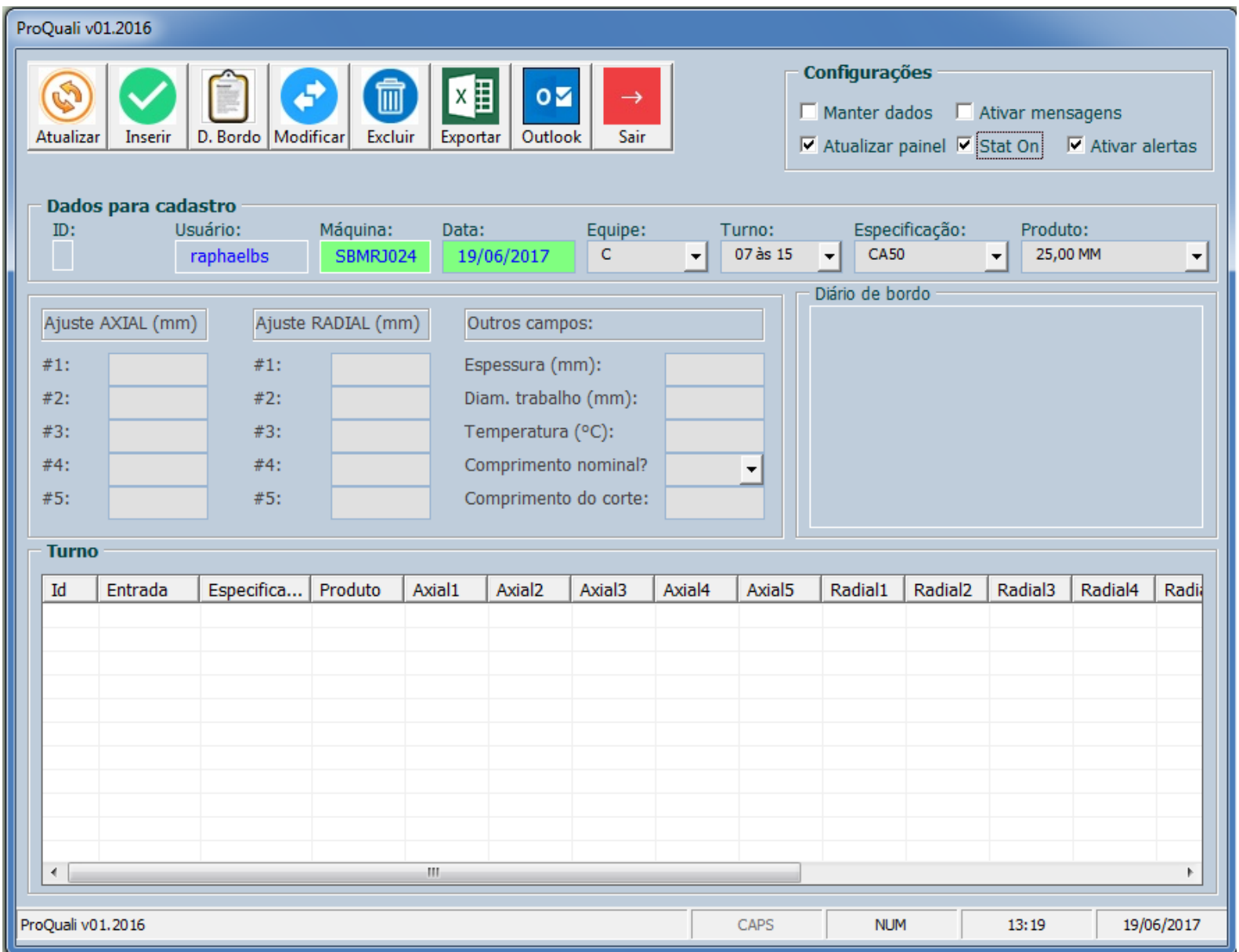

Figura 7. Formulário para cadastro das variáveis da endireitadeira de barras.

O ProQuali v01.2016 é a base do Programa da Qualidade (ProQuali) proposto pela equipe do projeto, sendo o início do processo, onde as informações são registradas e armazenadas no banco de dados para o controle online do processo e pesquisas futuras. Para estabelecer o próximo nível do Programa da Qualidade, ou seja, as análises e controle, foi desenvolvido o STATPro v01.2017, que é a ferramenta disponível para fazer todo o gerenciamento dos dados, quer por comando do usuário (que pode ser a equipe técnica ou operacional) ou por controle automático.

O STATPro v01.2017 é um programa de análises e relatórios que inclui:

- Análises estatísticas e de controle de processo;

- Análises de campanhas;

- Controle online do processo para o operador do laminador e inspetor da qualidade (postos 1 e 2 da figura 2).

Na figura 8 observa-se a tela de acesso do STATPro v01.2017. 


\section{Carta de Controle}

Carta I-AM

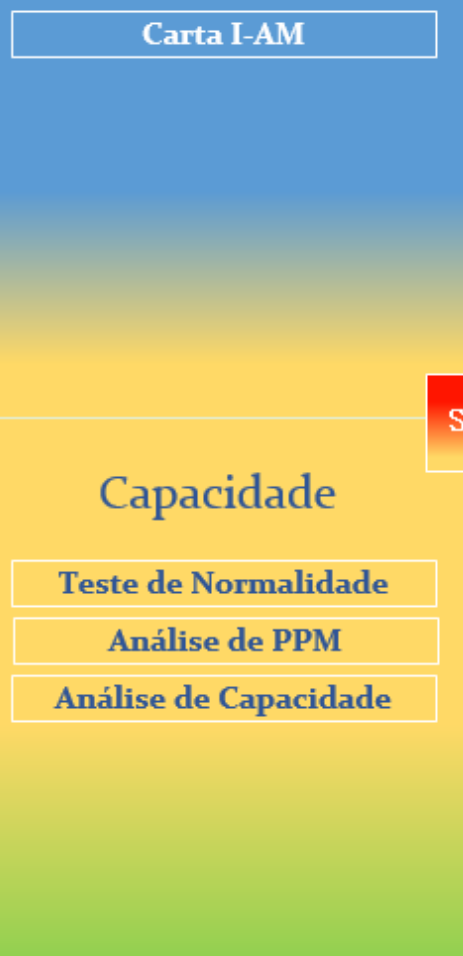

Figura 8.Tela inicial de acesso do STATPro v01.2017.

Na figura 9, observa-se o painel de controle online do laminador, que fica disponível durante todo o processo para o operador acompanhar as variáveis de controle conforme o produto laminador.

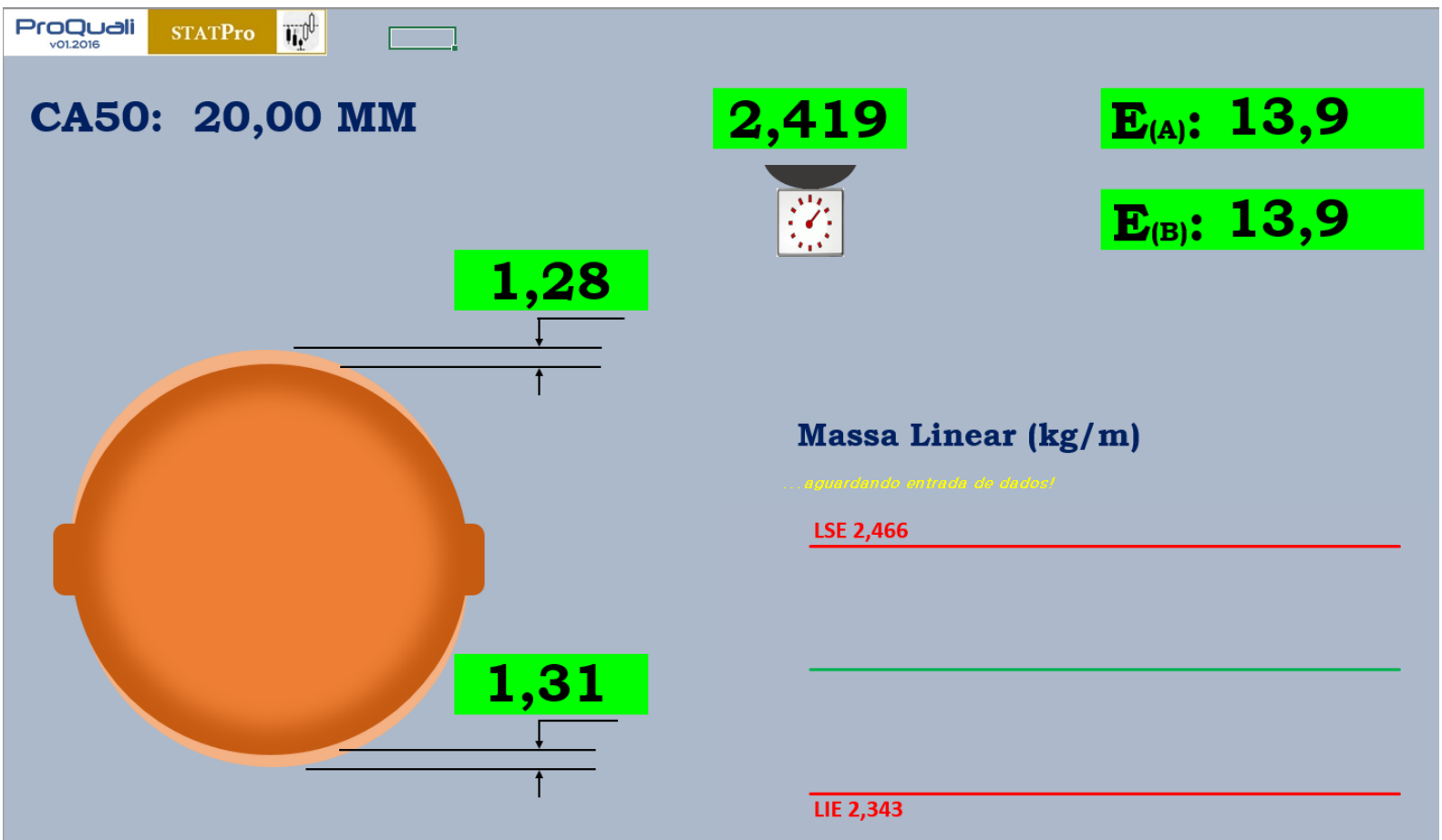

Figura 9. Painel de Controle Online para o laminador. 


\subsection{Verificar}

Após o desenvolvimento do ProQuali e testes de ajustes, disponibilizamos e acompanhamos com os operadores a versão do ProQuali v01.2016 para o laminador, inspeção online, laboratório de ensaios e endireitamento de barras.

Quando validamos a eficácia e organização dos dados no banco, finalizamos a etapa de programação do ProQuali v01.2016 e desenvolvemos o STATPro v01.2017 e o Painel de Controle Online para o laminador, conforme mostra a figura 9. A equipe verificou que com esse novo modelo de gestão da qualidade (ProQuali) os ganhos em análise, tomada de decisão e controle do processo foram mais eficientes e robustos com a utilização do ProQuali v01.2016 e STATPro v01.2017, permitindo atuar no processo de forma rápida.

\subsection{Padronização}

Estabelecemos o Programa da Qualidade com o uso do ProQuali v01.2016 e STATPro v01.2017. Após validar todas as etapas do projeto, treinamos todos os envolvidos no novo modelo de gestão e padronizamos o processo com base no ProQuali.

\section{RESULTADOS E DISCUSSÃO}

O principal resultado do ProQuali foi a capacidade de extrair e analisar uma grande massa de dados em um intervalo de tempo relativamente curto e fazer o controle online, possibilitando um controle mais eficiente e atuação nas variáveis de controle do processo antes da geração de produtos não conformes (NC).

Com ProQuali v01.2016, os dados foram organizados para dar ênfase em: gestão de processos, análises de falhas no processo, análises de campanhas, análise de reclamação de clientes, suporte para auditorias internas e externas, monitoramento online do processo e análises estatística. Também notamos uma rápida adaptação dos usuários com a interface simples. Com o STATPro v01.2016, o programa de análise estatística, a equipe conseguiu ganhos significativos no tempo de análise, conforme mostra a figura 10 que compara o tempo de análise mensal para a estabilidade e capacidade dos produtos laminados:

\begin{tabular}{|c|c|c|c|}
\hline Mês & Produtos & StatPro & Manual \\
\hline jan/17 & 49 & $11,61 \mathrm{~s}$ & $24,50 \mathrm{~h}$ \\
\hline $\mathrm{fev} / 17$ & 57 & $11,13 \mathrm{~s}$ & $28,50 \mathrm{~h}$ \\
\hline $\mathrm{mar} / 17$ & 48 & $10,72 \mathrm{~s}$ & $24,00 \mathrm{~h}$ \\
\hline $\mathrm{abr} / 17$ & 53 & $10,52 \mathrm{~s}$ & $26,50 \mathrm{~h}$ \\
\hline mai/17 & 54 & $10,84 \mathrm{~s}$ & $27,00 \mathrm{~h}$ \\
\hline
\end{tabular}

Figura 10. Ganho nas análises com o STATPro.

Na figura 10 é comparado o tempo de análise do STATPro v01.2016 com o tempo necessário para o modelo de gestão anterior ao Programa da Qualidade (ProQuali). Outros ganhos com o Programa da Qualidade, incluindo a integração entre os sistemas ProQuali v01.2016 e STATPro v01.2017 foram as evidências na melhoria dos indicadores do laminador, que auxiliou a equipe na análise e tomada de decisão. Esses ganhos foram mais expressivos nas campanhas de BRL em 2017 comparando com 2016: 
- Redução de $72 \%$ no número de geração de produtos não conformes;

- Aumento de $4,4 \%$ no índice de utilização;

- Aumento de 2,5\% no índice de rendimento metálico;

- Atuação preventiva no processo antes da geração de produtos não conforme ou instabilidade do processo.

\section{CONCLUSÃo}

O ProQuali é um projeto piloto que está sendo utilizado como modelo base para a busca contínua da melhoria no sistema de gestão da qualidade do Laminador para garantir a otimização das análises das variáveis de processo e produto.

O modelo foi bem aceito pelos operadores da área como uma melhoria na gestão da qualidade e do processo, possibilitando aos operadores do piso do laminador o acompanhamento online através dos gráficos de tendências dos dimensionais dos produtos. Devido a isto, a atuação do operador durante o processo passou a ser realizada de maneira preventiva, visando evitar às ocorrências de perdas, principalmente por geração de produtos não conformes. Também concluímos que 0 Programa da Qualidade pode ser replicado para outros laminadores, com os mesmos conceitos do ProQuali v01.2016 e STATPro v01.2017.

\section{AGRADECIMENTOS}

Nossos sinceros agradecimentos à Votorantim Siderurgia pela oportunidade de desenvolver um programa da qualidade que possibilitasse a otimização dos nossos resultados e uma rápida análise para tomadas de decisão sobre o processo de laminação. Com a mesma importância, também agradecemos à nossa gerência pelo apoio e incentivo durante a caminhada do ProQuali e aos nossos colegas e colaboradores que apoiaram o programa, aceitaram como uma oportunidade de melhora para as suas atividades e contribuíram com suas valiosas sugestões.

Um agradecimento especial aos membros do time de desenvolvimento do ProQuali pela perseverança e a busca contínua por boas práticas ao encarar os desafios encontrados durante as fases do Programa da Qualidade.

\section{REFERÊNCIAS}

1 Salvendy G. Handbook of Industrial Engineering: Technology and Operations Management. 3aㅡ ed. New York: John Wiley \& Sons, Inc.; 2001.

2 Bessant J, Caffyn S, Gilbert J, Haring R, Weeb S. Rediscovering continuous improvement. Technovation. Fevereiro 1994: V.14, n.1: 17-29.

3 Moura R. Qualidade simplesmente total: uma abordagem simples e prática da gestão da qualidade. Rio de Janeiro: Qualitymark; 1997. 